\title{
Bose-Bose Mixtures with Synthetic Spin-Orbit Coupling in Optical Lattices
}

\author{
Liang $\mathrm{He}^{1,3}$, Anchun $\mathrm{Ji}^{2}$, and Walter Hofstetter ${ }^{1}$ \\ ${ }^{1}$ Institut für Theoretische Physik, Goethe-Universität, 60438 Frankfurt/Main, Germany \\ ${ }^{2}$ Center for Theoretical Physics, Department of Physics, \\ Capital Normal University, Beijing 100048, China and \\ ${ }^{3}$ Institut für Theoretische Physik, Leopold-Franzens Universität Innsbruck, A-6020 Innsbruck, Austria
}

\begin{abstract}
We investigate the ground state properties of Bose-Bose mixtures with Rashba-type spin-orbit (SO) coupling in a square lattice. The system displays rich physics from the deep Mott-insulator (MI) all the way to the superfluid (SF) regime. In the deep MI regime, novel spin-ordered phases arise due to the effective Dzyaloshinskii-Moriya type super-exchange interactions. By employing the non-perturbative Bosonic Dynamical Mean-Field-Theory (BDMFT), we numerically study and establish the stability of these magnetic phases against increasing hopping amplitude. We show that as hopping is increased across the MI to SF transition, exotic superfluid phases with magnetic textures emerge. In particular, we identify a new spin-spiral magnetic texture with spatial period 3 in the superfluid close to the MI-SF transition.
\end{abstract}

PACS numbers: 67.85.-d, 37.10.Jk, 71.70.Ej

\section{INTRODUCTION}

Spin-orbit (SO) effects have been known in solid-state physics for a long time and have for example been observed in graphite more than four decades ago [1]. But their main effects were believed to simply split the spin degenerate band structures [2]. It is only until recently that both theory and experiment have revealed that strong spin-orbit coupling can give rise to dramatic qualitative effects, leading to distinct and novel phases of matter such as topological band insulators [3, 4$]$. This has motivated experimental developments in the field of quantum simulation with ultracold atoms, where tunable SO coupling and more generally synthetic non-abelian gauge fields are created in both neutral bosonic atoms [5-7] and fermionic atoms [8, 9] via Raman processes or by driven optical lattices [10].

This progress has stimulated interesting studies on the physics of SO coupled Bose-Bose mixtures subjected to an optical lattice [11 16], where the Mott insulator to superfluid phase transition [11, 12], magnetic order in the deep Mott insulator regime [12 15], and superfluid phases [11, 12, 14, 16] are investigated. In particular, in the strongly interacting regime, a super-exchange spin model with Dzyaloshinskii-Moriya (DM) type interactions [17, 18] can be derived by second-order perturbation theory. And various exotic spin-textures are predicted by classical Monte-Carlo simulations or spin-wave analysis of the effective spin model [12-15]. Similar spin textures induced by the DM-type interactions have also been found in various solid state materials and attracted great interest [19 21]. Moreover, it is interesting to notice that bosons in a certain class of frustrated lattices have similar dispersion relations as particles with isotropic Rashba SO coupling [22], which could indicate that similar exotic magnetic phases may also be relevant in these systems.

However, there remain fundamental open questions. Generally, the second-order super-exchange model only applies in the deep Mott regime, not to mention certain cases, e.g. in presence of geometric frustration, where it does not even apply in the deep Mott regime [23]. One highly relevant question for experiments aiming at direct observation of these exotic spin-textures at realistic temperature is whether they are stable against increasing hopping amplitude, in particular in the Mott regime close to the Mott insulator to superfluid (MI-SF) phase transition, where the second-order super-exchange model no longer holds true. Another important issue is that the two-particle interactions play a fundamental role in determining the properties of the superfluid phase in the vicinity of the MI-SF phase transition, where the spinorbit coupling can induce exotic superfluid phases.

In this work, we investigate these fundamental issues within the non-perturbative theoretical framework of Bosonic Dynamical Mean Field Theory (BDMFT) 2427]. We find that the exotic spin-textures are robust throughout the whole Mott regime, see Fig. 2, This is of practical importance for experimental observation of these phases at realistic temperatures. In the vicinity of the MI-SF transition, we show that exotic superfluid phases arise as a result of the interplay between onsite interaction and SO coupling, as shown in Fig. 3 In particular, we identify a new spin-spiral magnetic texture with spatial period 3 on the SF side near the MI-SF transition, see Fig. 3. b1.

\section{MODEL AND METHOD}

We consider two species of bosons with SO coupling loaded in a square optical lattice. For sufficiently low filling, we model the system by a two-component BoseHubbard model in the lowest band approximation, which reads

$$
H=-t \sum_{\langle i, j\rangle}\left(\psi_{i}^{\dagger} \mathcal{R}_{i j} \psi_{j}+\text { h.c. }\right)+\frac{1}{2} \sum_{i \sigma \sigma^{\prime}} U_{\sigma \sigma^{\prime}} \hat{a}_{i \sigma}^{\dagger} \hat{a}_{i \sigma^{\prime}}^{\dagger} \hat{a}_{i \sigma^{\prime}} \hat{a}_{i \sigma} .
$$


Here $\langle i, j\rangle$ denotes nearest-neighbor sites, $\psi_{i}^{\dagger} \equiv\left(\hat{a}_{i \uparrow}^{\dagger}, \hat{a}_{i \downarrow}^{\dagger}\right)$ with $\hat{a}_{i \sigma}^{\dagger}\left(\hat{a}_{i \sigma}\right)$ being bosonic creation (annihilation) operators of the two species labeled by pseudo-spin $\sigma=\uparrow, \downarrow$, on site $i$ in the Wannier representation. $t$ is the overall hopping amplitude and the matrix $\mathcal{R}_{i j}=\exp [i \vec{A}$. $\left.\left(\mathbf{r}_{i}-\mathbf{r}_{j}\right)\right]$, where $\vec{A}=\left(\alpha \sigma_{y}, \beta \sigma_{x}, 0\right)$ denotes a static, non-abelian gauge field which can be generated by using a two-photon Raman process [28]. Here, $\sigma_{x}, \sigma_{y}$ and $\sigma_{z}$ are the Pauli matrices. We shall consider the case $\beta=-\alpha$, which implies that the $\mathrm{SO}$ coupling is of Rashba type. In this case, the explicit form of $\mathcal{R}_{i j}$ reads: $\mathcal{R}_{i j}=\cos \alpha \cdot \mathbf{I} \pm i \sin \alpha \sigma_{y}$, for $\mathbf{r}_{i}-\mathbf{r}_{j}= \pm \hat{\mathbf{x}}$ and $\mathcal{R}_{i j}=\cos \alpha \cdot \mathbf{I} \mp i \sin \alpha \sigma_{x}$, for $\mathbf{r}_{i}-\mathbf{r}_{j}= \pm \hat{\mathbf{y}}$. We remark here that a similar type of SO coupling, which is a mixture of Rashba and Dresselhaus coupling, has been recently realized experimentally in trapped two-species bosonic quantum gases [7]. The second term in Eq. (1) describes the on-site interactions. We choose the intraspecies repulsion $U_{\downarrow \downarrow}=U_{\uparrow \uparrow} \equiv V$, and set the interspecies interaction $U_{\uparrow \downarrow}=U_{\downarrow \uparrow} \equiv U=\lambda V$ with a dimensionless parameter $\lambda$. In our investigation, we shall focus on the ground state properties of the system at total filling per site $\rho \equiv \sum_{i}\left\langle\hat{a}_{i \uparrow}^{\dagger} \hat{a}_{i \uparrow}+\hat{a}_{i \downarrow}^{\dagger} \hat{a}_{i \downarrow}\right\rangle / N_{\text {lat }}=1$, with $N_{\text {lat }}$ being the number of lattice sites.

To investigate the properties of the system in the full range of interactions, i.e. from the strong coupling deep MI regime all the way to the SF at weak coupling, we apply BDMFT [24 27], which is non-perturbative and can capture the local quantum fluctuations exactly. For exploring various possible exotic magnetic or superfluid phases which break the translational symmetry of the lattice, here we specifically employ real-space BDMFT (RBDMFT) [29], which generalizes BDMFT to a positiondependent self-energy and captures inhomogeneous quantum phases. Within this approach, the physics on each lattice site is determined from a local effective action obtained by integrating out all other degrees of freedom in the lattice model, excluding the lattice site considered. The local effective action is then represented by an Anderson impurity model [24 27]. We use exact diagonalization (ED) [30, 31] of the effective Anderson Hamiltonian with a finite number of bath orbitals to solve the local action. For the results presented in this work, $n_{\text {bath }}=4$ bath orbitals and a $12 \times 12$ lattice are chosen. Typical physical results are checked with a larger number of bath orbitals $\left(n_{\text {bath }}=5\right)$ and lattice size $(24 \times 24)$ and no qualitative difference are found. Details of the R-BDMFT method can be found in previous work [29].

\section{RESULTS}

\section{A. Mott-insulator regime}

In the deep MI regime for $U_{\sigma \sigma^{\prime}} \gg t$, the physics of the system can be captured by an effective spin model obtained by second order perturbation theory [12 15], which is given by

$$
H_{\mathrm{eff}}=\sum_{i, \delta=\hat{\mathbf{x}}, \hat{\mathbf{y}}}\left[\sum_{a=x, y, z} J_{\delta}^{a} S_{i}^{a} S_{i+\delta}^{a}+\mathbf{D}_{\delta} \cdot\left(\mathbf{S}_{i} \times \mathbf{S}_{i+\delta}\right)\right],
$$

where $S_{i}^{a} \equiv \psi_{i}^{\dagger} \sigma^{a} \psi_{i} / 2$ with $a=x, y, z$. The first and the second term denote the Heisenberg-type (H-type) and Dzyaloshinskii-Moriya type (DM-type) super-exchange coupling respectively. $J_{\delta}^{a}$ and $\mathbf{D}_{\delta}$ are the corresponding coupling strengths. The explicit form of $J_{\delta}^{a}$ and $\mathbf{D}_{\delta}$ are given by $J_{\hat{x}}^{x}=J_{\hat{y}}^{y}=-4 t^{2} \cos (2 \alpha) / \lambda V, J_{\hat{x}}^{y}=$ $J_{\hat{y}}^{x}=-4 t^{2} / \lambda V, J_{\hat{x}}^{z}=J_{\hat{y}}^{z}=-4 t^{2}(2 \lambda-1) \cos (2 \alpha) / \lambda V$, $\mathbf{D}_{\hat{x}}=-4 t^{2} \sin (2 \alpha) \hat{y} / \lambda V$ and $\mathbf{D}_{\hat{y}}=4 t^{2} \sin (2 \alpha) \hat{x} / \lambda V$. Since these two types of couplings favor qualitatively different types of magnetic orders, namely, $\mathrm{H}$-type couplings favor either ferromagnetic or anti-ferromagnetic type order while the DM-type couplings favor spiral type order, the interplay between them can drive the system into various exotic magnetically ordered states.

Instead of studying the effective exchange Hamiltonian (2), we directly simulate the original lattice boson Hamiltonian (11) within BDMFT. Our results in the deep MI regime are summarized in Fig. 11, which shows a phase diagram of the system at a fixed small hopping $t=0.001 U$ and an interaction ratio $\lambda=0.8$. When the SO coupling is weak $(|\alpha| \ll 1)$ the system is dominated by the $\mathrm{H}$-type exchange interactions. For $\lambda<1$ the spin-exchange in the $x y$-plane is larger than the one in the $z$-direction and ferromagnetic, hence the system favors an $x y$-ferromagnetic phase as expected. Upon further increasing the SO coupling, the DM-type interaction begins to play a role and drives a transition from the $x y$-ferromagnet to the spiral magnetic phases (red interval in the phase diagram in Fig. (1). Due to the limitation of finite system size in our simulations, we are not able to resolve possible incommensurate spiral order. For simulations on a $12 \times 12$ lattice presented in this paper, we are able to resolve commensurate spiral order of spatial period 12,6 and 4 lattice sites, denoted as spiral-12, spiral-6 and spiral-4 for simplicity. To be more specific, in the simulations on a $12 \times 12$ lattice, we observe a sequence of transitions from spiral-12 to spiral-6 to spiral-4 upon increasing SO coupling strength $\alpha$. Further increasing $\alpha$ drives the system from the spiral phase to a $3 \times 3$ skyrmion phase (blue interval in the phase diagram in Fig. (1).

Further increasing $\alpha$ drives another transition from the $3 \times 3$ skyrmion phase to a $2 \times 2$ spin vortex whose existence can be attributed to the spin-flip hopping process due to SO coupling. To make this point more specific, let us focus on the limit $|\alpha-\pi / 2| \ll 1$. From the explicit form of the hopping matrix element $\mathcal{R}_{i j}$, we can see that the conventional spin-conserving hopping is strongly suppressed in this case, i.e. particles on neighboring sites are mainly coupled by spin-flip hopping processes arising from SO coupling. This gives rise to an unconventional $\mathrm{H}$-type interaction in the effective spin Hamilto- 


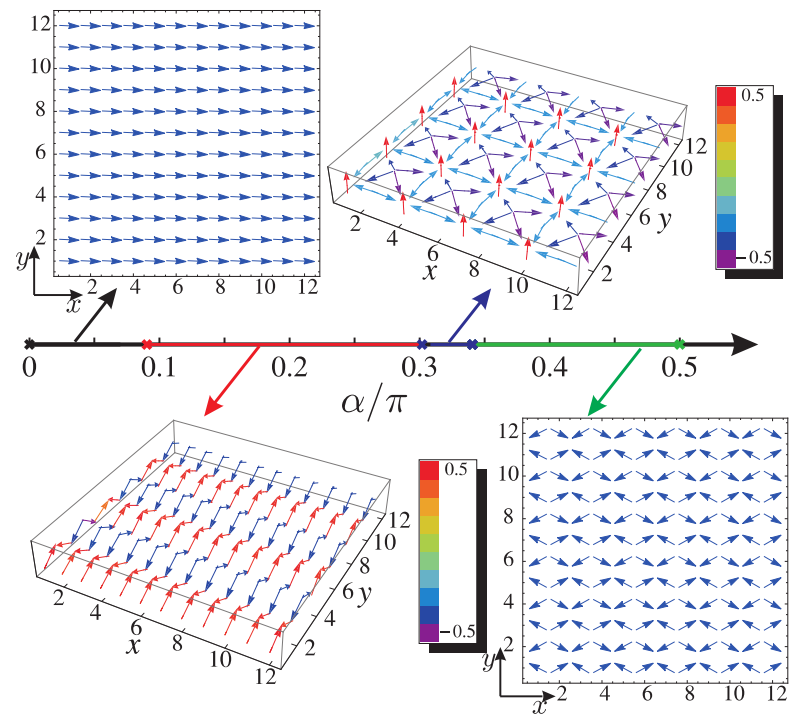

FIG. 1: (color online). Ground state phase diagram in the deep Mott-insulator regime $(t=0.001 U)$ in terms of Rashbatype SO coupling parameter $\alpha$ at a fixed interaction ratio $\lambda=0.8$ obtained by simulations on a $12 \times 12$ square lattice. Four types of phases, i.e. $x y$-ferromagnetic (black interval), spiral (red interval), $3 \times 3$ skyrmion lattice (blue interval), and $2 \times 2$ spin-vortex (green interval) phases are found. The $2 \mathrm{D}$ vector plots of the $x y$-ferromagnetic and the $2 \times 2$ spin-vortex phases correspond to the $x y$-magnetization distribution, since these two phases are co-planar. The $3 \mathrm{D}$ vector plots show the magnetization vector in $3 \mathrm{D}$ space, where the color of the arrow denotes the magnetization in $z$-direction.

nian with a spatially-isotropic antiferromagnetic interaction in $z$-direction, and exchange interactions which are anisotropic both in the spatial directions and spin orientations in the $x y$-plane. Since the H-type exchange couplings dominate over the DM-type ones in this regime, they determine the magnetic order. For $\lambda<1$ the spin interaction in the $x y$-plane is larger than the one in the $z$ direction, hence the system favors a co-planar magnetic order. As we can see from the explicit form of $J_{\delta}^{a}$ the exchange for the $x$ component is antiferromagnetic along the spatial $x$ direction, but ferromagnetic along the $y$ direction. This indicates that the $x$-component of the spins favors to align parallel along the (spatial) $y$ direction, while anti-parallel along the $x$ direction. Similarly, one can see that the $y$-component of the spins favors to align parallel along the $x$ direction but anti-parallel along the $y$ direction. As a result, this unconventional H-type interaction gives rise to a $2 \times 2$ spin vortex configuration in the $x y$-plane, as shown in the right bottom part of Fig. 1 .

Although we thus confirm numerically that the exchange Hamiltonian Eq. (22) is a good description in the deep MI regime, due to the perturbative nature of the effective spin model, it is important to investigate whether these exotic magnetic phases are stable against quantum fluctuations for large hopping $t / U$. For this

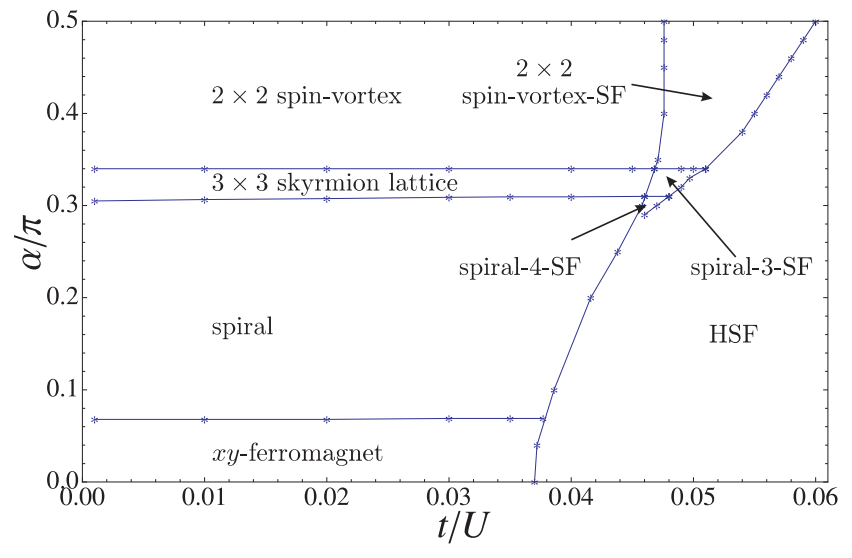

FIG. 2: (color online). Ground state phase diagram in terms of SO coupling strength $\alpha$ and hopping amplitude $t$ at a fixed interaction ratio $\lambda=0.8$, obtained by simulations on a $12 \times 12$ square lattice. There are four magnetic phases in the Mottinsulator regime: $x y$-ferromagnet, spiral phase, $3 \times 3$ skyrmion lattice phase and $2 \times 2$ spin-vortex phase. In the $\mathrm{SF}$ regime three phases exist, namely the homogenous superfluid (HSF), a superfluid with $2 \times 2$ spin-vortex magnetic texture $(2 \times 2$ spin-vortex-SF), and a superfluid with spiral magnetic texture with spatial period of 3 lattice sites (spiral-3-SF).

purpose, we calculate the full $\alpha-t$ phase diagram within BDMFT, as shown in Fig. 2. We find that, although the validity of the effective spin model (2) is restricted to the deep MI regime, the exotic spin-textures are robust throughout the whole Mott regime up to small shifts of the boundaries between the different phases. These results are of practical importance in experiments aiming at observing these exotic magnetic phases, since the effective exchange interaction strengths are proportional to $t^{2}$, yielding higher critical temperatures $\left(\sim k_{B}^{-1} t^{2} / V\right.$ with $k_{B}$ being the Boltzmann constant) of the magnetic phases for larger values of $t$.

\section{B. MI-SF transition and exotic superfluid phases with magnetic textures}

As it is well known, the MI-SF transition occurs when the bandwidth ( $\sim 8 t$ for square lattice) is comparable to the interaction strength $U$. However, in Fig. 2 we find an $\alpha$-dependence of the SF-MI transition boundary. This can be qualitatively understood within the slaveboson picture [12]. The spinful lattice boson annihilation (creation) operator $\hat{a}_{i \sigma}\left(\hat{a}_{i \sigma}^{\dagger}\right)$ in the original lattice boson Hamiltonian (11), can be decomposed into the bosonic annihilation (creation) operators of "spinon" $\hat{f}_{i \sigma}\left(\hat{f}_{i \sigma}^{\dagger}\right)$ and "chargon" $\hat{c}_{i}\left(\hat{c}_{i}^{\dagger}\right)$, with $\hat{a}_{i \sigma}=\frac{1}{\sqrt{\hat{n}_{i}}} \hat{c}_{i} \hat{f}_{i \sigma}, \hat{n}_{i}=\hat{c}_{i}^{\dagger} \hat{c}_{i}$, and the constraint $\sum_{\sigma} \hat{f}_{i \sigma}^{\dagger} \hat{f}_{i \sigma}=\hat{c}_{i}^{\dagger} \hat{c}_{i}$ projected to the physical Hilbert space. Then, the lattice-boson Hamiltonian (11) assumes another representation, denoted as $H_{\mathrm{SC}}$, in terms of annihilation (creation) operators of spinon and 

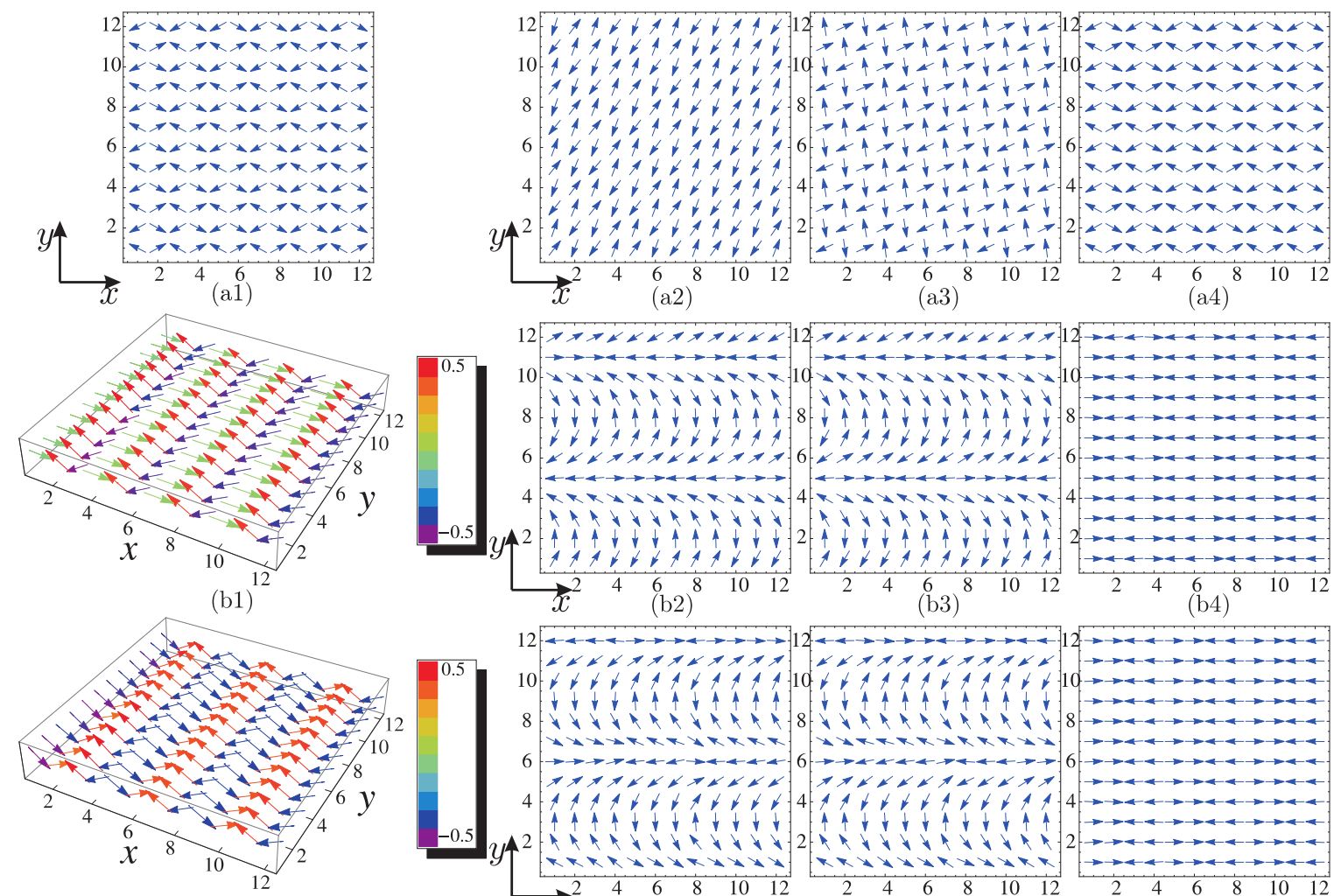

(c1)
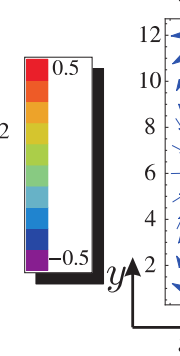

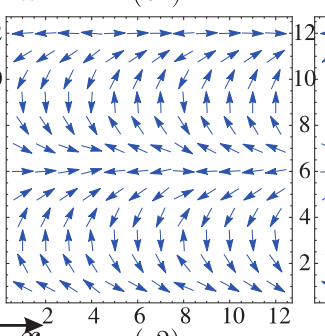

$(\mathrm{c} 2)$

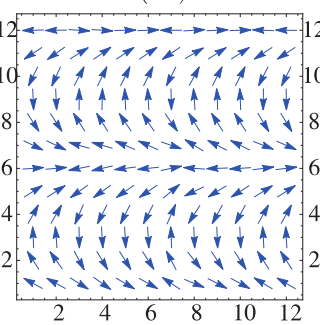

(c3)

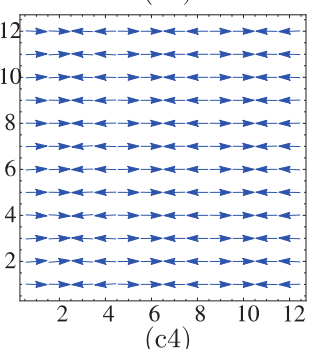

$(\mathrm{c} 4)$

FIG. 3: (color online). Typical superfluid phases with non-uniform magnetic order. From up to down, different rows of plots correspond to $2 \times 2$ spin-vortex SF phase, spiral-3 SF phase and spiral-4 SF phase respectively. From left to right different columns corresponds to the real-space distribution of magnetization, $\arg \left(\left\langle a_{i \uparrow}\right\rangle\right), \arg \left(\left\langle a_{i \downarrow}\right\rangle\right) \operatorname{and} \arg \left(\left\langle a_{i \uparrow}\right\rangle^{*}\left\langle a_{i \downarrow}\right\rangle\right)$. For the $2 \times 2$ spin-vortex SF phase since the magnetization is co-planar, only a $2 \mathrm{D}$ vector plot is shown. The $3 \mathrm{D}$ vector plots correspond to the magnetization vector in $3 \mathrm{D}$ space and the color of the arrow denotes the magnetization in $z$-direction.

chargon,

$$
\begin{aligned}
H_{\mathrm{SC}}= & -t\left(\frac{1}{\sqrt{\hat{n}_{i} \hat{n}_{j}}} \hat{c}_{i}^{\dagger} \hat{c}_{j} \hat{\Phi}_{i}^{\dagger} \mathcal{R}_{i j} \hat{\Phi}_{j}+\text { h.c. }\right) \\
& -\mu \sum_{i} \hat{\Phi}_{i}^{\dagger} \hat{\Phi}_{i}-\sum_{i} \mu_{i}^{c}\left(\hat{c}_{i}^{\dagger} \hat{c}_{i}-\hat{\Phi}_{i}^{\dagger} \hat{\Phi}_{i}\right) \\
& +\frac{U}{2} \sum_{i}\left(\hat{n}_{f, i, \uparrow}^{2}+\hat{n}_{f, i, \downarrow}^{2}+2 \lambda \hat{n}_{f, i, \uparrow} \hat{n}_{f, i, \downarrow}\right),
\end{aligned}
$$

where $\hat{\Phi}_{i}^{\dagger} \equiv\left(\hat{f}_{i \uparrow}^{\dagger}, \hat{f}_{i \downarrow}^{\dagger}\right)$ and $\mu_{i}^{c}$ is introduced to keep the local constraint satisfied on average $\left\langle\sum_{\sigma} \hat{f}_{i \sigma}^{\dagger} \hat{f}_{i \sigma}\right\rangle=\left\langle\hat{c}_{i}^{\dagger} \hat{c}_{i}\right\rangle$. By making a mean-field approximation to the spinon, i.e. $\hat{\Phi}_{i} \rightarrow \Phi_{i}$ with $\Phi_{i}$ 's being $c$-number valued vectors, and keeping only the leading order contribution of the "spinon" to the physics of the chargon, the effective Hamiltonian of the chargon degree of freedom, denoted as $H_{\mathrm{C}}^{\mathrm{eff}}$, assumes the form of the conventional Bose-Hubbard model

$$
H_{\mathrm{C}}^{\mathrm{eff}}=-\sum_{\langle i, j\rangle}\left(\tilde{t}_{i j} \hat{c}_{i}^{\dagger} \hat{c}_{j}+h . c .\right)+\frac{U}{2} \sum_{i} \hat{c}_{i}^{\dagger} \hat{c}_{i}^{\dagger} \hat{c}_{i} \hat{c}_{i}
$$

where $\tilde{t}_{i j}=t \Phi_{i}^{\dagger} \mathcal{R}_{i j} \Phi_{j}$ can be regarded as the "spinonrenormalized" hopping amplitude of the chargon. Since the MI-SF transition is driven by the chargons, from Eq. (44) we directly see that different mean-field configurations of the spinon, which correspond to different magnetic configurations, give rise to different renormalized chargon hopping amplitudes $\tilde{t}_{i j}$, and hence make the MISF transition boundary $\alpha$-dependent.

From the phase diagram in Fig. 2, we see that SF phases with magnetic textures emerge near the MI-SF transition. In particular, we observe a new spin-spiral magnetic texture with spatial period of 3 (spiral-3) in the SF phase near the MI-SF transition [see Fig. 3(b1)]. A qualitative picture is that the chargon degree of freedom undergoes drastic changes across the MI-SF transition, and the feedback of the chargon to the spinon degree of freedom modifies the magnetic textures in the SF phase pronouncedly. From our simulation results, we observe that the phase angle distribution of the superfluid order parameters has complex structures [see Fig. 3(a2), (b2), (c2), (a3), (b3), and (c3)]. Interestingly, for the superfluid with $2 \times 2$ spin-vortex magnetic structure, superfluid vortices are observed as well [see Fig. 33(a3)]. Moreover, it is interesting to see that the relative phase $\Delta \theta_{i} \equiv \theta_{i \downarrow}-\theta_{i \uparrow}$ between the two superfluid components has a simple texture which coincides with the corresponding magnetic 
texture on the $x-y$ plane [see Fig. [3(a4), (b4), and (c4)]. Qualitatively, this can be understood within the slaveboson picture. The condensate order parameter in the slave-boson representation reads $\phi_{\sigma} \equiv\left\langle a_{i \sigma}\right\rangle=\left\langle c_{i} f_{i \sigma}\right\rangle$. Assuming that the correlations between chargon and spinon are small, we approximate $\phi_{i \sigma} \approx\left\langle c_{i}\right\rangle\left\langle f_{i \sigma}\right\rangle$. Then the relative phase between the two superfluid components is given by $\arg \left(\phi_{i \uparrow}^{*} \phi_{i \downarrow}\right)=\arg \left(\left\langle f_{i \uparrow}\right\rangle^{*}\left\langle f_{i \downarrow}\right\rangle\right)$, together with the fact that the magnetization on the $x-y$ plane is given by $\left\langle S_{i}^{x}\right\rangle=\Phi_{i}^{\dagger} \sigma_{x} \Phi_{i} / 2=\left|\left\langle f_{i \uparrow}\right\rangle\left\langle f_{i \downarrow}\right\rangle\right| \cos \left(\arg \left(\left\langle f_{i \uparrow}\right\rangle^{*}\left\langle f_{i \downarrow}\right\rangle\right)\right)$ and $\left\langle S_{i}^{y}\right\rangle=\Phi_{i}^{\dagger} \sigma_{y} \Phi_{i} / 2=\left|\left\langle f_{i \uparrow}\right\rangle\left\langle f_{i \downarrow}\right\rangle\right| \sin \left(\arg \left(\left\langle f_{i \uparrow}\right\rangle^{*}\left\langle f_{i \downarrow}\right\rangle\right)\right)$, we see that the texture of the relative phase between the two superfluid components is indeed the same as the magnetic texture in the $x-y$ plane.

\section{CONCLUSION}

We have investigated ground state properties of BoseBose mixtures with Rashba-type spin-orbit (SO) coupling loaded in a square optical lattice, within the nonperturbative theoretical framework of BDMFT. The sys- tem shows rich spin physics in both the Mott-insulator and the superfluid regime. We found that the exotic magnetic phases are robust in the whole MI regime. In the proximity of the MI-SF transition, exotic superfluid phases with magnetic textures arise as a result of the interplay between onsite interaction and SO coupling. In particular, we identify a new spin-spiral magnetic texture with spatial period 3 on the SF side near the MI-SF transition. These results are of practical importance for experimental observation of these exotic spin texture and novel SF phases.

\section{Acknowledgments}

L. H. thanks X. Zhang, D. Cocks, M. Buchhold, S. Diehl, R. Li, and Q. Sun for useful discussions. This work was supported by the Deutsche Forschungsgemeinschaft DFG via Sonderforschungsbereich SFB/TR 49 and Forschergruppe FOR 801. A. C. Ji was supported by NCET and NSFC under grants No. 11474205.
[1] G. Dresselhaus and M.S. Dresselhaus, Phys. Rev. 140, A401 (1965).

[2] Solid State Physics, N. W. Ashcroft and N. D. Mermin, Brooks Cole (1976).

[3] M. Hasan and C. Kane, Rev. Mod. Phys. 82, 3045 (2010).

[4] X. L. Qi and S. C. Zhang, Rev. Mod. Phys. 83, 1057 (2011).

[5] Y. J. Lin, R. L. Compton, A. R. Perry, W. D. Phillips, J. V. Porto, and I. B. Spielman, Phys. Rev. Lett. 102, 130401 (2009).

[6] Y. J. Lin, R. L. Compton, K. Jiménez-García, J. V. Porto, and I. B. Spielman, Nature 462, 628 (2009).

[7] Y. J. Lin, K. Jiménez-García, and I. B. Spielman, Nature 471, 83 (2011).

[8] P. J. Wang, Z. Q. Yu, Z. K. Fu, J. Miao, L. H. Huang, S. J. Chai, H. Zhai, and J. Zhang, Phys. Rev. Lett. 109, 095301 (2012).

[9] L. W. Cheuk, A. T. Sommer, Z. Hadzibabic, T. Yefsah, W. S. Bakr, and M. W. Zwierlein, Phys. Rev. Lett. 109, 095302 (2012).

[10] J. Struck, C. Ölschläger, M. Weinberg, P. Hauke, J. Simonet, A. Eckardt, M. Lewenstein, K. Sengstock, and P. Windpassinger, Phys. Rev. Lett. 108, 225304 (2012).

[11] T. Graß, K. Saha, K. Sengupta, and M. Lewenstein, Phys. Rev. A 84, 053632 (2011).

[12] W. S. Cole, S. Z. Zhang, A. Paramekanti, and N. Trivedi, Phys. Rev. Lett. 109, 085302 (2012).

[13] J. Radic, A. Di Ciolo, K. Sun, and V. Galitski, Phys. Rev. Lett. 109, 085303 (2012).

[14] Z. Cai, X. Zhou, and C. Wu, Phys. Rev. A 85, 061605(R) (2012).

[15] M. Gong, Y. Y. Qian, V. W. Scarola, and C. W. Zhang,
arXiv:1205.6211 (2012).

[16] Y. Qian, M. Gong, V. W. Scarola, and C. W. Zhang, arXiv:1312.4011 (2013).

[17] I. Dzyaloshinsky, J. Phys. and Chem. Sol. 4, 241 (1958).

[18] T. Moriya, Phys. Rev. 120, 91 (1960).

[19] D. Pesin and L. Balents, Nature Phys. 6, 376 (2010).

[20] S. Heinze, K. von Bergmann, M. Menzel, J. Brede, A. Kubetzka, R. Wiesendanger, G. Bihlmayer, and S. Blügel, Nature Phys. 7, 713 (2011).

[21] S. Banerjee, O. Erten, and M. Randeria, Nature Phys. 9, 626 (2013).

[22] T. A. Sedrakyan, L. I. Glazman, and A. Kamenev, Phys. Rev. B 89, 201112 (2014).

[23] L. He, Y. Q. Li, E. Altman and W. Hofstetter, Phys. Rev. A 86, 043620 (2012).

[24] K. Byczuk and D. Vollhardt, Phys. Rev. B 77, 235106 (2008).

[25] A. Hubener, M. Snoek, and W. Hofstetter, Phys. Rev. B 80, 245109 (2009).

[26] W. J. Hu and N. H. Tong, Phys. Rev. B 80, 245110 (2009).

[27] P. Anders, E. Gull, L. Pollet, M. Troyer, and P. Werner, Phys. Rev. Lett. 105, 096402 (2010).

[28] K. Osterloh, M. Baig, L. Santos, P. Zoller, and M. Lewenstein, Phys. Rev. Lett. 95,010403 (2005).

[29] Y. Q. Li, M. R. Bakhtiari, L. He, and W. Hofstetter, Phys. Rev. B 84, 144411 (2011).

[30] M. Caffarel and W. Krauth, Phys. Rev. Lett. 72, 1545 (1994).

[31] Q.-M. Si, M. J. Rozenberg, G. Kotliar, and A. E. Ruckenstein, Phys. Rev. Lett. 72, 2761 (1994). 